\title{
Bowel Injury and Insidious Pneumoperitoneum after Lateral Lumbar Interbody Fusion
}

\author{
Eui Seung Hwang ${ }^{1}$, Kook Jong Kim ${ }^{2}$, Choon Sung Lee ${ }^{3}$, Mi Young Lee ${ }^{3}$, \\ So Jung Yoon ${ }^{3}$, Jae Woo Park ${ }^{4}$, Jae Hwan $\mathrm{Cho}^{3}$, Dong-Ho Lee ${ }^{3}$ \\ ${ }^{1}$ College of Arts and Sciences, Emory University, Atlanta, GA, USA \\ ${ }^{2}$ Department of Orthopaedic Surgery, Chungbuk National University College of Medicine, Cheongju, Korea \\ ${ }^{3}$ Scoliosis Center, Asan Medical Center, University of Ulsan College of Medicine, Seoul, Korea \\ ${ }^{4}$ Department of Orthopaedic Surgery, Gangneung Asan Hospital, Gangneung, Korea
}

Study Design: Retrospective review of prospectively collected cases.

Purpose: To report bowel injury cases and determine the incidence and risk factors of insidious pneumoperitoneum after lateral lumbar interbody fusion (LLIF).

Overview of Literature: Minimally invasive LLIF is considered a safe surgical approach with a low risk of complications. Visceral injury after LLIF is rare and, to our knowledge, no studies on pneumoperitoneum after LLIF have been performed. Bowel injury is a catastrophic complication, but the clinical signs may not be apparent. After we encountered two cases of bowel injury after LLIF, we decided to perform computed tomography of the abdomen and pelvis (APCT) after surgery for all patients who underwent LLIF.

Methods: A total of 90 patients underwent APCT within 48 hours of surgery. Medical records were reviewed to determine each patient's age, sex, body mass index, medical and surgical histories, characteristics of LLIF procedures, and subjective symptoms and abnormal findings in the physical examination related to acute abdomen after surgery. Various parameters were compared between patients with and without pneumoperitoneum.

Results: Bowel injuries were identified in the first two patients and five patients (5.5\%) were diagnosed with pneumoperitoneum only on APCT. We found that the greater the number of fused segments, the higher the incidence of postoperative bowel injury and/or pneumoperitoneum. The incidence was significantly high when the L2-3 level was included in the LLIF surgery.

Conclusions: Pneumoperitoneum after LLIF indicates damage to the peritoneum and the presence of bowel injury that may lead to peritonitis. However, it is difficult to distinguish pneumoperitoneum and/or bowel injury from general abdominal pain after surgery because patients may present with a wide range of symptoms. We recommend that APCT be routinely performed after LLIF surgery in order to promptly identify pneumoperitoneum and bowel injury.

Keywords: Bowel injury; Pneumoperitoneum; Lateral lumbar interbody fusion

\section{Introduction}

Lateral lumbar interbody fusion (LLIF) has been a popu- lar modality for the treatment of degenerative disorders and deformities. It is a modification of the conventional retroperitoneal approach to the lumbar spine and is usual-

Received Apr 14, 2021; Revised Jun 6, 2021; Accepted Jun 26, 2021

Corresponding author: Kook Jong Kim

Department of Orthopaedic Surgery, Chungbuk National University Hospital, 776 1(il)sunhwan-ro, Heungdeok-gu, Cheongju 28644, Korea

Tel: +82-43-269-6114, Fax: +82-43-274-8719, E-mail: spine.cbnuh.or.kr 
ly performed in a minimally invasive fashion to overcome drawbacks of traditional approaches [1-3]. Minimally invasive LLIF has the advantages of a small skin incision, rapid recovery, and direct access to the disk space without peritoneal or paraspinal muscular injury. Wider and higher cages can be placed onto the strong peripheral cortical bone with the LLIF technique than with the posterior approach, minimizing the risk of cage subsidence, especially in elderly patients with osteoporotic spine, and maximizing the correction power in deformity correction surgeries [4].

Despite its minimally invasive nature, several approachrelated complications have been reported, including neurological injury, psoas muscle weakness, pseudohernia, and vascular injury [5]. Although the incidence of bowel injuries due to LLIF surgery is minimal, the injuries can be fatal in the absence of early diagnosis and treatment [612].

The authors have frequently utilized the LLIF approach for the treatment of various spinal conditions. Although several reports on bowel injury after the anterior approach to the spine have already been published in the literature [6-12], owing to the low incidence of such complications, we failed to realize the seriousness of this risk until we encountered two such cases. Therefore, we decided to perform a computed tomography (CT) scan of the abdomen and pelvis (APCT) for every patient and analyzed the incidence and risk factors for postoperative pneumoperitoneum and bowel injury. The purpose of this study was to report on bowel injury cases and determine the incidence of and risk factors for insidious pneumoperitoneum after LLIF.

\section{Materials and Methods}

Since we introduced the LLIF technique for treating degenerative lumbar disorders and deformities in July 2016, 140 LLIF surgeries were performed at the department of orthopaedic surgery of Asan Medical Center until October 2018. Although we encountered various approachand cage-related complications, bowel injury had not been observed after surgery until October 2018, when we observed injuries in two patients (cases 1 and 2). Therefore, we decided to perform an APCT for every patient who underwent LLIF in order to promptly identify insidious bowel injury. This is a retrospective review of the APCT results of our patients during 1 year.
A total of 90 patients underwent APCT on postoperative day 1. APCT was performed within 48 hours of surgery in all patients except for case 1 . The medical records of all study participants were reviewed to determine age, sex, body mass index (BMI), characteristics of LLIF procedures, and medical and surgical histories. Data on postoperative conditions were also documented, including subjective symptoms and abnormal findings in the physical examination related to acute abdomen. APCT scans were reviewed by radiologists specializing in abdominal and pelvic disorders. The study was approved by the ethical review committee of Asan Medical Center. The requirement for informed consent from individual patients was omitted because of the retrospective design of this study.

Statistical analyses were performed to compare patients with and without pneumoperitoneum. Continuous variables such as age, BMI, operation time, estimated blood loss, and cage height were compared using MannWhitney $U$-test. Categorical variables such as sex, history of abdominal/pelvic surgery, direction of approach, and operated levels were compared using Fisher's exact test. The numbers of operated segments were compared using a linear by linear association test. All statistical analyses were performed with IBM SPSS Statistics for Windows ver. 21.0 (IBM Corp., Armonk, NY, USA). All p-values $<0.05$ were considered statistically significant.

\section{Results}

Among 90 patients who underwent APCT after LLIF surgery, bowel injuries were identified in the first two patients, and pneumoperitoneum only in five patients (5.5\%). No obvious visceral injury was observed in the APCT scans of the five patients. Of these five patients, one patient had no subjective symptoms or abnormal findings in the physical exam; the other four patients had mild abdominal discomfort without abnormal findings in the physical exam.

There were no statistically significant differences in the characteristics between patients with and without pneumoperitoneum (Table 1). Of the seven patients with bowel injury and/or pneumoperitoneum, only one patient had a history of abdominal and/or pelvic surgery (case 1). The right approach was used in one patient and the left approach in six patients. All patients except case 2 underwent fusion at more than three levels (Table 2). We 
Table 1. Comparison of parameters between patients with no abnormal findings in computed tomography of the abdomen and pelvis (group 1) and patients with postoperative pneumoperitoneum and/or bowel injury (group 2)

\begin{tabular}{lccc}
\hline Characteristic & Group 1 & Group 2 & $p$-value \\
Age (yr) & 68.8 & 70.4 & 0.786 \\
Sex (male/female) & & & 0.603 \\
$\quad$ Male & 14 & 2 & \\
$\quad$ Female & 69 & 5 & \\
\hline Body mass index (kg/m²) & 26.4 & 26.0 & 0.381 \\
Surgical history in abdomen/pelvis (\%) & 18.1 & 14.3 & 0.638 \\
\hline Direction of approach & & & 0.444 \\
$\quad$ Right & 6 & 1 & \\
$\quad$ Left & 77 & 6 & \\
\hline Operation time (min) & 326.1 & 258.3 & 0.302 \\
\hline Estimated blood loss (mL) & 927.5 & 964.3 & 0.464 \\
Cage height (mm) & 12.6 & 12.7 & 0.591 \\
\hline
\end{tabular}

Table 2. Incidence of postoperative bowel injury and/or pneumoperitoneum after lateral lumbar interbody fusion according to the number of segments operated

\begin{tabular}{lrcr} 
No. of segments & Group 1 & Group 2 & $p$-value \\
\hline 1 & 26 & 0 & 0.012 \\
2 & 26 & 1 & \\
\hline 3 & 19 & 3 & \\
\hline 4 & 11 & 3 & \\
\hline 5 & 1 & 0 \\
\hline
\end{tabular}

Table 3. Incidence of postoperative pneumoperitoneum and/or bowel injury after LLIF according to the operated level

\begin{tabular}{lccc} 
Level & No. of patients $^{\text {a) }}$ & Pneumoperitoneum & $p$-value \\
L1-2 & 6 & 0 & 0.606 \\
L2-3 & 36 & 6 & 0.037 \\
L3-4 & 59 & 7 & 0.104 \\
L4-5 & 68 & 7 & 0.266 \\
L5-S1 & 15 & 3 & 0.140 \\
\hline
\end{tabular}

The bold type is considered statistically significant.

LLIF, lateral lumbar interbody fusion.

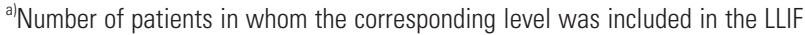
surgery.

found that the greater the number of fused segments, the higher the incidence of postoperative bowel injury and/or pneumoperitoneum $(p=0.012)$. The incidence was significantly high when the L2-3 level was included in the LLIF surgery (Table 3 ).

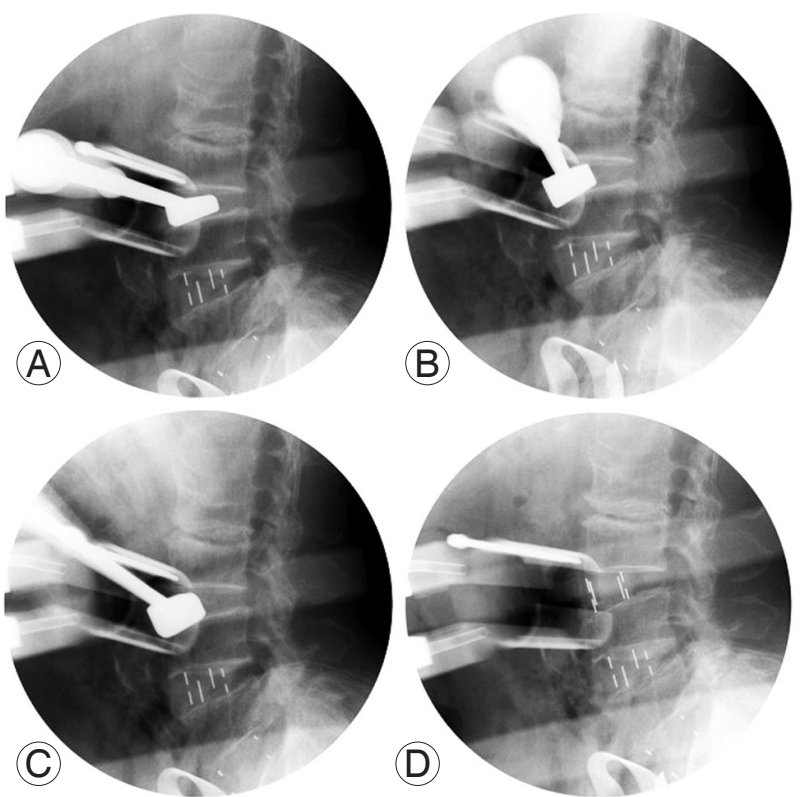

Fig. 1. (A-D) Intraoperative fluoroscopic images of case 1 during lateral lumbar interbody fusion of $\mathrm{L} 3-4$

\section{Case 1}

A 75-year-old female presented with severe lower-back pain, left leg pain, and neurogenic claudication that were not relieved by long-term conservative treatment. The patient had degenerative lumbar scoliosis, multilevel spinal stenosis, and isthmic spondylolisthesis at L5-S1. Medical comorbidities included hypertension, diabetes, and cerebrovascular disease. She had a history of skin cancer resection and hysterectomy. Magnetic resonance imaging (MRI) and CT scan of the spine showed no obvious issues of concern for a retroperitoneal approach.

Minimally invasive LLIF was performed using polyetheretherketone (PEEK) cages to the left side at L2-34-5-S1 for deformity correction and indirect decompression, and posterior pedicle screw fixation and fusion were added in 1-day surgery. It was difficult to secure the surgical field for L3-4 disc space preparation and cage placement due to protruded peritoneum; as a result, the L4 endplate was inadvertently injured and the trial instrument tended to move forward (Fig. 1). No definitive evidence of peritoneal and bowel injury was found upon inspection during removal of the retractor after completing the procedure. On postoperative day 5 , fever persisted and subcutaneous emphysema developed around the surgical site. Although the patient complained of mild abdominal discomfort, on physical examination there was no defini- 
tive abnormal sign suggesting bowel injury.

The APCT scan revealed extensive pneumoperitoneum, pneumopericardium, pneumomediastinum, and subcutaneous emphysema (Fig. 2). Although definitive findings suggesting bowel injury were not evident on the CT scan, an emergency exploratory laparotomy was performed,

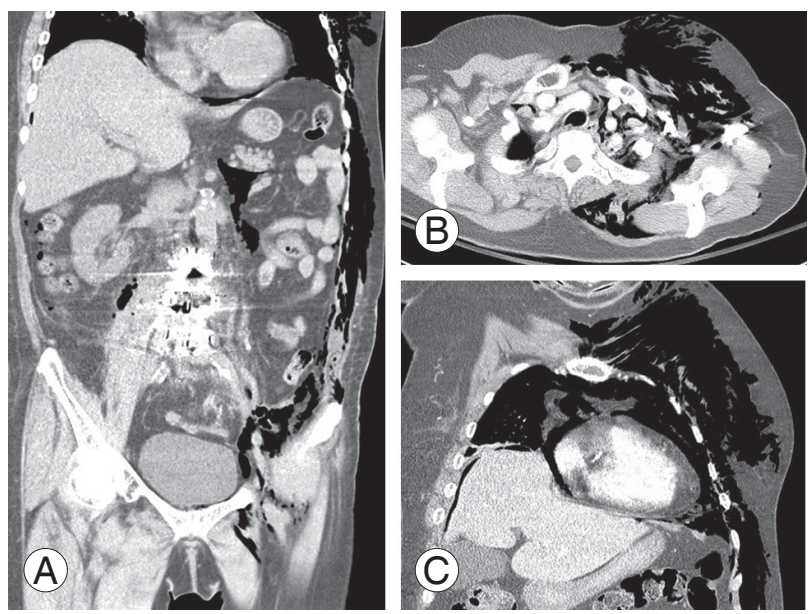

Fig. 2. (A-C) Postoperative chest and abdomen computed tomography images of case 1.
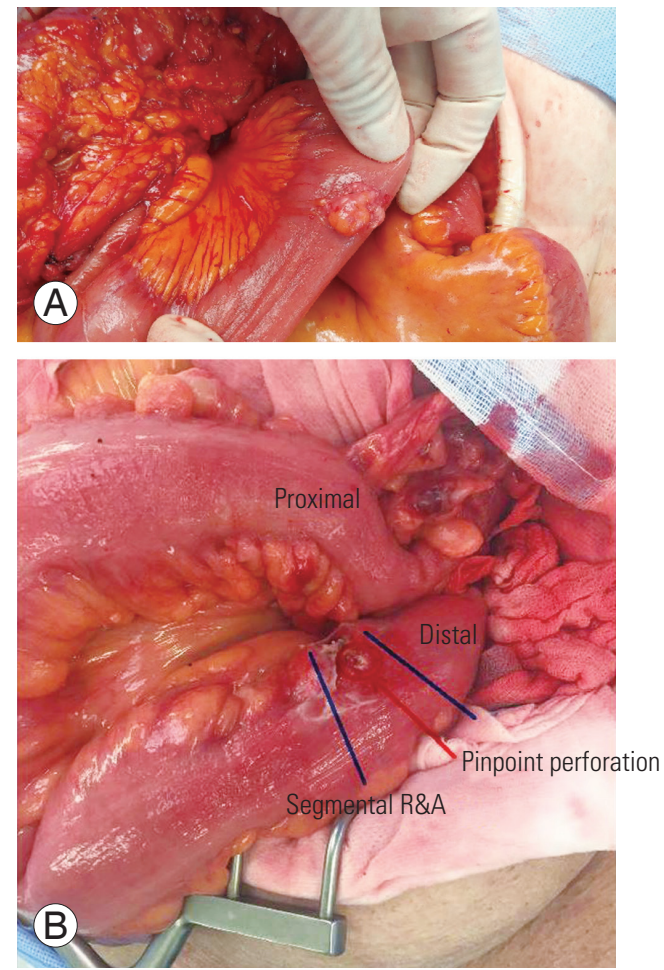

Fig. 3. (A) Intraoperative photograph of mid-descending colon perforation during explorative laparotomy in case 1. (B) Intraoperative photograph of sigmoid colon perforation during explorative laparotomy in case 2. Segmentral R\&A, segmental resection and anastomosis. and a $2 \times 2 \mathrm{~cm}$-sized perforation of the mid-descending colon and surrounding peritoneal defect were observed (Fig. 3). Part of the descending colon including the perforation site was resected and temporary colostomy was performed. The colostomy was repaired after 3 months and the patient recovered uneventfully without any further complications.

\section{Case 2}

A 78-year-old male patient presented with severe lowerback pain and right sciatica. Although he had undergone posterior lumbar interbody fusion surgery at L4-5 at another hospital 3 weeks previously, the pain and disability had progressed to the extent that he could not walk for a while. He had no history of prior abdominal and pelvic surgery. Radiographs and MRI scans of the lumbar spine showed screw loosening and cage subsidence, which aggravated his stenosis. Postoperative infection was also suspected and the level of C-reactive protein in his blood was high.

Posterior surgical exploration was immediately performed, but there was no definitive evidence of infection in the operative field and no causative organism was identified. After 2 weeks of empirical antibiotic treatment, new cage placement by the LLIF technique and posterior revision instrumentation were performed. The pre-existing loose cage was removed through the same approach. L3-4 was also included in the fusion level because of substantial spinal stenosis. There was no definitive evidence of peritoneal and bowel injury during the procedure.

On postoperative day 1 , the patient was admitted to the intensive care unit for neck swelling and dyspnea. Although neck CT and APCT scans showed extensive
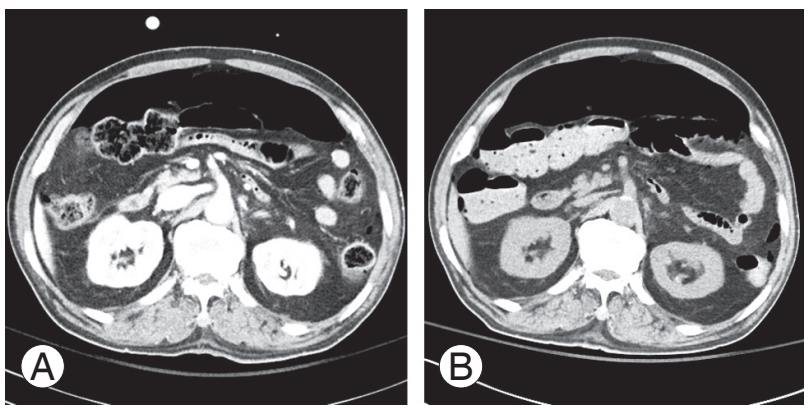

Fig. 4. (A) Image of computed tomography of the abdomen and pelvis of case 2 on postoperative day 1. (B) Image of computed tomography of the abdomen and pelvis of case 2 on postoperative day 5 demonstrating aggravation of pneumoperitoneum. 


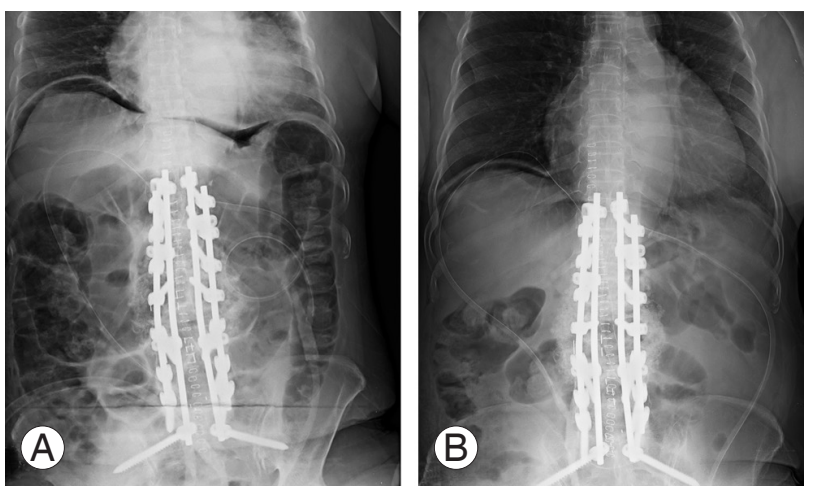

Fig. 5. Simple erect abdomen radiograph of a patient with postoperative pneumoperitoneum. (A) On postoperative day 2, substantial amount of gas shadow was observed. (B) On postoperative day 5, improvement of pneumoperitoneum was confirmed.

subcutaneous emphysema from the neck to the operation site and pneumoperitoneum (Fig. 4), he did not complain of abdominal pain, and signs suggesting acute abdomen were not definitive. There was no evidence of bowel injury and perforation focus in the APCT. The patient's general condition and neck swelling subsequently improved and he was transferred to the general ward on postoperative day 2. However, abdominal pain and distention developed on postoperative day 5 , and follow-up APCT scan showed aggravated pneumoperitoneum (Fig. 5). Emergency laparotomy was performed, and pinpoint perforation was found at the end of the sigmoid colon. Hence, a part of the sigmoid colon including the perforation site was resected and temporary colostomy was performed. The colostomy was repaired after 3 months, and the patient uneventfully recovered without any further complications.

\section{Discussion}

Although bowel injury after LLIF is very rare, it is a potentially life-threatening complication. A few studies have documented a small number of cases of bowel perforation after LLIF [9-12]. Its exact incidence is not known. Uribe et al. [5] reported that $11(0.08 \%)$ of 13,004 patients had visceral complications after extreme lateral interbody fusion in a survey study. In addition, in their retrospective study, Rustagi et al. [11] found that three patients had bowel injury after a total of 590 transpsoas lumbar interbody fusion surgeries, resulting in a procedure-specific incidence of $0.51 \%$. We encountered two consecutive cases of bowel perforation after LLIF around the same time and found that bowel injury may not be easily diagnosed during or after surgery because the relevant symptoms and signs are vague and nonspecific. Therefore, we decided to routinely perform APCT for every patient who underwent LLIF surgery; eventually, five patients were found to have postoperative pneumoperitoneum. This means that the peritoneum is damaged more frequently than expected, even if bowel injury is absent.

Uribe et al. [5] reported that comorbidities were common in patients who had bowel injuries, with each patient having at least one major preoperative comorbid factor. We paid attention to the history of abdominal and pelvic surgery rather than comorbidities because patients with severe comorbidities are usually not scheduled for spine surgery, and intra-abdominal adhesion is a critical factor for peritoneal and bowel injury during minimally invasive retroperitoneal approaches. In case 1, the patient with bowel injury had a history of hysterectomy, whereas in case 2, the patient had no surgical history. Of the five patients with postoperative pneumoperitoneum only, none had a history of abdomen and pelvis surgery. On the other hand, 14 patients had a history of abdomen and pelvis surgery among the 83 patients whose postoperative APCT scans showed no evidence of pneumoperitoneum and bowel injury. We surmise that other factors play more important roles than comorbidities and surgical history in the development of bowel and peritoneal injury after LLIF.

An anatomical study evaluating the risk of colon injury in extreme lateral approaches to the lumbar spine indicated the presence of a high risk of colon injury at the L2-3 and L3-4 levels [13]. In the current study, the incidence of pneumoperitoneum and/or bowel injury was significantly high when the L2-3 level was included in the LLIF surgery. However, it is not known at which level peritoneal tear and/or bowel injury developed because all patients with postoperative pneumoperitoneum and/or bowel injury underwent multilevel LLIF and peritoneal rent could not be identified on the APCT scans. In case 1 , the descending colon injury was probably attributable to the L3-4 procedure. In case 2, the sigmoid colon was probably injured during the L4-5 procedure.

Uribe et al. [5] suggested that a two-incision approach may decrease the risk of visceral injury, as $70 \%$ of the patients with visceral injury were treated using a single lateral incision. They did not report the number of levels treated. At our institution, we almost always use a two- or three-incision approach for multilevel LLIF. Importantly, all patients with bowel injury or pneumoperitoneum ex- 
cept case 2 underwent more than three levels of LLIF. According to our statistical analysis, the number of segments included was significantly associated with the incidence of pneumoperitoneum and/or bowel injury. In case 1, an unexpected event of forward slippage of the trial instrument during the L3-4 procedure occurred, and in case 2, the pre-existing cage was extracted through a minimally invasive small incision. Attention to technical issues is crucial for avoiding injuries to intra-abdominal structures. Complete development of the retroperitoneal space is essential and the tube dilator must be firmly pressed against the psoas muscle and spine to prevent the intra-abdominal structures from moving underneath the dilator. If intraabdominal contents intrude between the blades of the tube dilator, they must be protected with gauze packing or another shielding device. At the end of the procedure, the operative field should be carefully examined during removal of the dilator to make sure that there is no evidence of bowel and/or peritoneal injuries and active bleeding.

However, bowel injury cannot be completely prevented by these measures. High clinical suspicion is of utmost importance to avoid devastating postoperative outcomes. While Rustagi et al. [11] reported that abdominal pain, distention, and fever were the most common findings in cases of visceral injury, most of the patients in our study complained of nonspecific and vague symptoms and signs. Abdominal pain and fever are common events after a retroperitoneal approach and spinal fusion. We recommend that all patients undergo APCT within at least 48 hours after LLIF, and if pneumoperitoneum is found without any evidence of bowel injury, serial simple sitting radiographs of the abdomen must be obtained to verify gradual decrease of free air and relevant symptoms.

The radiation exposure with APCT is higher than that with chest or head CT scans due to the large number of radiosensitive organs in the field of view [14]. Although the authors suggest that performing APCT for early detection of bowel injury after LLIF surgery may be justified, surgeons must recognize the need to balance such benefits with the possible risks of radiation exposure. Furthermore, patients must be informed of the need for the examination and the associated radiation risks.

The current study has some limitations. For example, the supporting data are insufficient to establish the risk factors for bowel injury after LLIF. Although APCTs were consecutively performed in all patients who underwent LLIF surgery after encountering the first bowel injury, this study is retrospective and the number of patients is small. Further prospective studies including a large number of patients are required and spine surgeons planning LLIF procedures need to be alert to avoid bowel injury, which is a catastrophic complication.

\section{Conclusions}

Although the occurrence of bowel injury after LLIF is rare, it can result in catastrophic complications if not promptly treated. The symptoms and signs suggesting acute abdomen may not be apparent even in patients with bowel perforation. In addition, a greater number of fused segments is related to a higher incidence of postoperative bowel injury and/or pneumoperitoneum. Therefore, we recommend that APCT be routinely performed after LLIF surgery in order to identify pneumoperitoneum and evidence of bowel injury.

\section{Conflict of Interest}

No potential conflict of interest relevant to this article was reported.

\section{References}

1. Ozgur BM, Aryan HE, Pimenta L, Taylor WR. Extreme lateral interbody fusion (XLIF): a novel surgical technique for anterior lumbar interbody fusion. Spin J 2006;6:435-43.

2. Hah R, Kang HP. Lateral and oblique lumbar interbody fusion-current concepts and a review of recent literature. Curr Rev Musculoskelet Med 2019;12:30510.

3. Khajavi K, Shen A, Lagina M, Hutchison A. Comparison of clinical outcomes following minimally invasive lateral interbody fusion stratified by preoperative diagnosis. Eur Spine J 2015;24 Suppl 3:322-30.

4. Hsieh MK, Chen LH, Niu CC, Fu TS, Lai PL, Chen WJ. Combined anterior lumbar interbody fusion and instrumented posterolateral fusion for degenerative lumbar scoliosis: indication and surgical outcomes. BMC Surg 2015;15:26.

5. Uribe JS, Deukmedjian AR. Visceral, vascular, and wound complications following over 13,000 lateral interbody fusions: a survey study and literature review. Eur Spine J 2015;24 Suppl 3:386-96. 
6. Epstein NE. Review of risks and complications of extreme lateral interbody fusion (XLIF). Surg Neurol Int 2019;10:237.

7. Walker CT, Farber SH, Cole TS, et al. Complications for minimally invasive lateral interbody arthrodesis: a systematic review and meta-analysis comparing prepsoas and transpsoas approaches. J Neurosurg Spine 2019:1-15.

8. Fujibayashi S, Kawakami N, Asazuma T, et al. Complications associated with lateral interbody fusion: nationwide survey of 2998 cases during the first 2 years of its use in Japan. Spine (Phila Pa 1976) 2017;42:1478-84.

9. Paterakis KN, Brotis AG, Paschalis A, Tzannis A, Fountas KN. Extreme lateral lumbar interbody fusion (XLIF) in the management of degenerative scoliosis: a retrospective case series. J Spine Surg 2018;4:610-5.
10. Tormenti MJ, Maserati MB, Bonfield CM, Okonkwo DO, Kanter AS. Complications and radiographic correction in adult scoliosis following combined transpsoas extreme lateral interbody fusion and posterior pedicle screw instrumentation. Neurosurg Focus 2010;28:E7.

11. Rustagi T, Yilmaz E, Alonso F, et al. Iatrogenic bowel injury following minimally invasive lateral approach to the lumbar spine: a retrospective analysis of 3 cases. Global Spine J 2019;9:375-82.

12. Balsano M, Carlucci S, Ose M, Boriani L. A case report of a rare complication of bowel perforation in extreme lateral interbody fusion. Eur Spine J 2015;24 Suppl 3:405-8.

13. Yilmaz E, Iwanaga J, Moisi M, et al. Risks of colon injuries in extreme lateral approaches to the lumbar spine: an anatomical study. Cureus 2018;10:e2122.

14. Tsapaki V, Rehani M, Saini S. Radiation safety in abdominal computed tomography. Semin Ultrasound CT MR 2010;31:29-38. 